\title{
Robust Representation of Shape in a Grey Parrot (Psittacus erithacus)
}

Irene M. Pepperberg ${ }^{\mathrm{a}, \mathrm{b}, *}$, Ken Nakayama ${ }^{\mathrm{a}}$

${ }^{a}$ Department of Psychology, Harvard University, Cambridge, MA, 02138, USA

${ }^{\mathrm{b}}$ Department of Psychology, Brandeis University, Waltham, MA 02454, USA

Keywords: Grey parrot, subjective contours, illusory contours, Kanizsa figures, avian cognition, avian perception, amodal completion, modal completion

\section{HIGHLIGHTS}

- Parrot is trained to identify 3D objects in using English speech.

- Without further training, parrot generalizes from 3D to 2D figures.

- Parrot labels partially occluded and Kanizsa figures using English speech.

* Corresponding Author

Dr. Irene M. Pepperberg

Department of Psychology

William James Hall

Harvard University

33 Kirkland Street

Cambridge, MA 02138

Ph. 617-495-2852

impepper@fas.harvard.edu

impepper@media.mit.edu 


\section{Introduction}

Data from many types of studies are used to claim that nonhuman subjects demonstrate various forms of higher order cognition (see, e.g., Wasserman \& Zentall, 2006; Zentall \& Wasserman, 2012). However, with few exceptions (e.g., 'absence' and a zero-like concept; Pepperberg \& Brezinsky, 1991; Pepperberg \& Gordon, 2005), such studies do not involve much in the way of strong generalization or transfer of concepts across domains, the latter being one of the few clear markers of higher order cognition (Rozin, 1976). Specifically, in most studies with nonhumans, subjects are taught basic concepts by rote, under a formal reward schedule, otherwise known as operant conditioning; although subjects can acquire highly refined distinctions such as motion direction, identity versus non-identity, or the ability to discriminate complex visual patterns, stimuli used in transfer tests to examine the generalization of such abilities rarely differ significantly from those used for training. This format is colloquially known as "teaching to the test." It is, however, an imperfect way to demonstrate broader knowledge (Oche, 2012). Notably, nonhuman subjects in these paradigms may fail when given strong transfer tests (Peissig et al., 2005). Strong generalization, the ability to perform under the widest circumstances without additional training, provides a proven evaluative metric for this broader knowledge in the fields of education (Gober, Baker, \& Sao Pedro, 2011) and even machine learning (e.g., Luis, Sucar \& Morales, 2010). Thus, a wider demonstration of this phenomenon would solidify claims for the robustness of nonhuman abilities with respect to learning and cognition.

Specifically, if we think of the most robust generalization/transfer as one that shows the most invariance of ability over the greatest range of image transformations, then we need to explore and test extremes. Prior studies (e.g., Pepperberg, 2006; Pepperberg \& Carey, 2012; Pepperberg \& Gordon, 2005) demonstrated that a Grey parrot could perform such generalizations and transfers with respect to number concepts, but the bird was tested in a more limited manner with respect to shape. That subject, named Alex (now deceased), had been trained via the model/rival (M/R) technique (see below) to understand the concept of shape (e.g., Pepperberg, 
1999), but was limited to three-dimensional (3D) regular polygons, transferring from uncolored 3D wood polygons of a single size to $3 \mathrm{D}$ polygons of various materials, sizes and colors. Could another Grey parrot demonstrate invariance over a much wider range of testing conditions, going for example, from 3D objects to 2D drawings? Furthermore, expanding our study to the phenomenon of occluded objects and subjective contours provides an unusually favorable situation for testing strong transfer, because training and testing images can be made to differ in more numerous and varied ways.

Objects are often not fully visible in many everyday scenes; partially covered by other objects, they are still easily seen and identified. Kanizsa $(1955,1979)$ described this most common behavior as amodal completion. Amodal completion is an ecologically valid and ubiquitous task. Despite the missing lower right corner, the square in Figure 1a is quickly recognized as a square. It is not seen as a figure from which a piece has been removed. Kanizsa also identified the process of modal completion, where objects are seen that are not accompanied by luminance changes at their boundaries. Otherwise known as subjective contours, they are visible despite the lack of any luminance transition as in the image in Figure 1b. Nakayama, He, and Shimojo (1995), using psychophysical tests, showed that such processes are likely localized within the visual system insofar as purely visual tasks such as motion perception, texture segregation, attentional allocation, and object recognition depend critically on these processes. Furthermore, neurophysiological recordings from single neurons by von der Heydt and colleagues (von der Heydt, Peterhans, \& Baumgartner, 1984; Zhou, Friedman, \& Von Der Heydt, 2000; Bakin, Nakayama \& Gilbert, 2000), demonstrated that neurons in the visual cortex show responses indicating the existence of such processes. Because the problems posed by occlusion and lack of luminance transitions across object boundaries are possibly universal across visual systems (see Nakayama et al., 1995), it would not be surprising if modal and amodal completion were evident in a very wide range of nonhuman species.

[Insert Figure 1 about here]

However, actually showing these abilities unambiguously with behavioral tests has not been 
easy. Results from many studies are consistent with various nonhuman subjects responding appropriately to $2 \mathrm{D}$ objects that are visible to humans as partially occluded or as being partly represented in their outline form by subjective contours (e.g., DiPietro, Wasserman, \& Young, 2002; Kanizsa et al., 1993; Horridge, Zhang, \& O’Connell, 1992; Martin-Malivel, 2011; Regolin \& Vallortigara, 1995; Van Hateren, Srinivasan, \& Wait,1990). However, all of these studies are subject to a variety of alternative interpretations. For example, subjects in these studies may have responded with respect to local cues, mass/number, or stimulus generalization (see Discussion). The point of the current study, therefore, was to avoid tests with results that could have alternative explanations. and recreate a situation more like that given to humans, who in most cases are given a small number of trials involving several different stimuli and simply asked to label what they see.

The present study thus has two advantages. First, our Grey parrot, Griffin, unlike subjects in other laboratories - with the exception of bonobos studied by Nagasaka and colleagues (Nagasaka, Brooks, \& Wasserman, 2010) - grew up in a very rich environment, more similar to that of preschool children. Thus he saw and manipulated real-world 3D objects, of all forms, materials, and colors, both in full view and occluded, and his exposure to these objects was protracted, over a period of over 16 years, before the study commenced. Such experience may be a prerequisite for carrying out the tasks being studied. Second, like Alex (deceased), he had learned English labels for various objects, foods, colors, shapes, and numbers, so that he could vocally describe, exactly, the items in his environment. Few nonhumans are capable of such behavior. Alex had, for example, responded to the Müller-Lyer illusion in a vocal manner consistent with that of humans (Pepperberg, Vicinay, \& Cavanagh, 2008). Griffin's ability to respond vocally, choosing from a multiplicity of labels, thus provides a unique opportunity to compare human and nonhuman data on completion tasks.

Experiments were therefore designed to answer the following queries: Can a Grey parrot (a) recognize 2D stimuli (e.g., as in Fig. 1) after having been trained on 3D objects? (b) perform identification tasks using novel images to show the presence of amodal and modal completion? (c) recognize nonstandard variations of the regular polygons he has learned to label? In other words, 
could the parrot perform these recognition tasks under very different circumstances from training, and with no training on the specific test stimuli? As noted above, such procedures are fairly standard for human subjects, but have not been fully implemented for nonhumans (note, however, Stephan, Wilkinson, \& Huber, 2013 for some level of trained 3D-2D transfer in pigeons).

\section{Experiment}

\subsection{Subject}

Griffin, a male Grey parrot (Psittacus erithacus), 16 years old at the beginning of the study, had been the subject of cognitive and communicative studies since he was 7.5 wks old (e.g., Pepperberg et al., 2013; Pepperberg \& Shive, 2001; Pepperberg \& Wilcox, 2000; Pepperberg, Willner, \& Gravitz, 1997). Testing locations and living conditions, when neither testing nor training were in progress, are described in Pepperberg and Wilkes (2004). Food and water were available at his vocal request at all times. In this study he used his abilities to produce and comprehend labels for various colors (red \{rose $\}$, blue, green, yellow, orange, purple) and shapes (regular polygons labeled "1-", "2", "3-", "4-", "6-", "8-corner"). Shapes on which he was trained were predominantly of wood or plastic, approximately 3-5 $\mathrm{cm}$ along the longest edge. Occasionally, shapes were made of flannel or paper, but because they were cut-outs, held between human's fingers and often rotated during presentation, and Griffin's reward was the opportunity to chew the items, they still retained a threedimensional quality (Fig. 2). He could also identify, with standard English number labels, sets of clicks, thus demonstrating a variety of quantification skills. At the beginning of the current study, his accuracy on identifying colors, shapes, materials and numbers generally averaged about 80-85\% (e.g., Pepperberg \& Wilcox, 2000; Pepperberg \& Wilkes, 2004).

[Insert Figure 2 about here]

\subsection{Initial Training}

The primary instructional procedure, described in detail elsewhere (Pepperberg, 1981, 1999) and known as model/rival or M/R training, is based primarily on methods developed by Todt (1975) 
and Bandura (1971). It involves three-way social interactions among two humans and a parrot to demonstrate the targeted vocal behavior. The parrot watches and listens as one trainer presents objects and queries the other trainer about them (e.g., "What's here?", "What color?", "What shape?"), giving praise and transferring the named object to the human partner to reward correct answers. Incorrect responses are punished by scolding and temporarily removing items from sight. Thus the second human is both a model for the parrot's responses and its rival for the trainer's attention, and illustrates consequences of errors. The model must try again or talk more clearly if the response was deliberately incorrect or garbled; that is, the model is subject to corrective feedback, which the bird observes. Furthermore, roles of trainer and model are exchanged so that the parrot sees that either can be the questioner or the respondent. The parrot is included in interactions, being queried and rewarded for successive approximations to correct responses; training is adjusted to its performance level. If a bird is inattentive or accuracy regresses, trainers threaten to leave.

To ensure the closest possible link between labels or concepts to be learned and their appropriate referent, $\mathrm{M} / \mathrm{R}$ training uses only intrinsic reinforcers: Reward for uttering " $\mathrm{X}$ " is $\mathrm{X}$, the object to which the label or concept refers (Fig. 3). Earlier unsuccessful attempts to teach birds to communicate with humans used extrinsic rewards: a single food neither relating to, nor varying with, the label or concept being taught (see Pepperberg, 1999), which confounded the label of the targeted exemplar or concept with that of the food reward. Initial use of labels as requests also demonstrates that uttering labels has functionality; later, birds learned "I want X," to separate requesting and labeling (Pepperberg, 1988) and to enable them to request preferred rewards while learning labels for items they had little interest in obtaining.

\section{[Insert Figure 3 about here]}

\subsection{Feasibility of Using 2D Stimuli}

Prior to this study, Griffin was never tested or trained with stimuli depicted on paper. All previous studies were with real objects: wooden and plastic triangles, squares, etc. Could we presume that Griffin would react to 2D depictions of real objects in an appropriate manner? Would he, when 
presented with a printed depiction of a square, identify it as such (“4-corner”)? Before testing him on amodal and modal completion, we needed to determine whether this was the case. Otherwise, failure to identify such test stimuli could be attributed to Griffin's inability to transfer to this medium.

To examine Griffin's abilities, we needed a two-dimensional depiction of a shape that would not interfere with the amodal and modal completion tests. One solution would be to simply present Griffin with a simple shape on a piece of paper, for example, a square. However, our stimuli for modal and amodal completion tests were more complex than a single object (look ahead to Fig. 5); we thus needed assurance that Griffin could focus on the appropriate target. Our solution was to present him with a fully completed shape (a square) occluding a disk which he had not been taught to identify (Fig. 4).

\section{[Insert Figure 4 about here]}

The timing to begin such testing was somewhat fortuitous, coming during a period of parrot "noncompliance", one aspect of which was perseveration: repetition of one response reflexively (i.e., staying with the answer to a previous query, even if the topic of questioning had changed; see Pepperberg \& Carey, 2012 for similar behavior in Alex; note related behavior, attributed to boredom, in keas, Gajdon Amann, \& Huber, 2011, and crows, Izawa \& Watanabe, 2011). Novelty can break such streaks; we thus decided to introduce the 2D stimuli described above (NB: colors of the square varied trial-to-trial to prevent habituation).

For 12 consecutive trials, Griffin labeled these 2D stimuli as "6-corner", much as he had for all queries over the previous several days. On the thirteenth trial, however, he responded "4-corner", suggesting that this noncompliant streak was broken and that he could be now tested with the modal and amodal completion stimuli (described below).

\section{$2.42 D$ Test Stimuli}

To test for amodal completion, we asked Griffin to label various colored regular polygons occluded .by black circles (e.g., asking "what shape blue?" as in Fig. 5c). Griffin had not been trained to label either circles or the color black making it unlikely that he would attempt to label them. We 
also used other black shapes as occluders, (e.g., Fig. 51), in order to see if being able to label the occluders would distract him from the task at hand. To fully test that Griffin was responding appropriately to occluded figures, it was also important to ask him to identify shapes that were not occluded. For example, compare Figure 5c with Figure 5h. At an image level the two figures are identical but only in Figure 5c is there the possibility of amodal completion, allowing us to see a triangle. In Figure 5h, this is not possible and what is seen is a figure that is no longer a triangle but one with four corners. As such, we predicted that Griffin would respond to Figure 5c as triangle, "3corner", but to Figure 5h as "4-corner". These latter non-occluded figures (5 in number, one for each of the \#-cornered objects being tested) are called detached probes ${ }^{1}$. An oval or circular shape was cut around every figure so the shape of the paper (“4-corner") would not be a factor in his response.

[Insert Figure 5 about here]

For modal completion — the task to identify shapes bounded by subjective contours-Kanizsa figures were constructed using black 'pac-men' to form regular polygons on colored paper. Controls ("probes", one or two for each of the \#-cornered shapes) involved placing additional circles or "pacmen' near the Kanizsa figure so Griffin could not simply quantify black objects (e.g., Fig. 5j). Nine such probes were used here. Stimuli were produced on a color laser printer, and here a random amorphous non-polygonal shape was cut out around the target stimuli so that the shape of the paper (“4-corner") would not be a factor in his response (see Fig. 5). The colors used were those tested in a previous study (Pepperberg et al., 2008) to ensure that Griffin would interpret the label for these printed colors correctly. ${ }^{2}$ Although Griffin could label octagons, these figures were not tested because we could not devise appropriately-sized stimuli comparable to the other possible shapes. A display of the full set of stimuli are given in Supplementary Material A.

Our choice to present only a few "probe" and "detached probe" trials, interspersed amongst the other test stimuli, was based on the criterion that, to confirm evidence of modal and amodal completion, the subject should respond appropriately to these trials on their first presentation so that no training could possibly be involved. Most experiments in the nonhuman literature use repeated 
presentation of identical probes for testing (e.g., Nagasaka \& Wasserman, 2008; Nakamura, Watanabe, Betsuyaku, \& Fujita, 2011), and either reward the subject for all probe trials (potentially encouraging guessing) or, after decreasing primary rewards to a set percentage similar to the proportion of probe trials, for none of the probe/test trials (potentially discouraging possible correct attempts). In some cases, testing is stopped in subjects that decrease in accuracy in the familiar (training) trials that continue to be presented during testing so they can be retrained (DiPietro et al., 2002). As experience may aid nonhuman subjects in solving amodal completion tasks (DiPietro et al., 2002, Nagasaka et al., 2010), such additional training likely increases the chance of success. To avoid any possible issues of familiarity, training, or encouraging either guessing or discouraging correct attempts, we decided that the strongest test would be to observe what our subject did on a single presentation of each possible probe. Note that the number of such probe trials in our study was not particularly small (13\% of amodal trials and $21 \%$ of modal trials), given that most experiments with nonhuman subjects intersperse approximately $11-25 \%$ of test trials within training stimuli (e.g., Fujita \& Giersch, 2005; Nagasaka \& Wasserman, 2008).

Note, too, that all of Griffin's trials were actually test trials, as he had never seen any of these stimuli prior to this study. To ensure that no trials of any type were administered consecutively (which could encourage learning), stimuli presentations were randomized with respect to modal/ amodal task, shape, size, and color, and all such trials were intermixed with trials on other types of tasks (see below). No specific stimulus (confluence of color/shape/size/arrangement of occluders or pac-men) was ever presented in more than one trial, although a shape query would be repeated for these different arrangements, but never for more than 5-6 times in these differing configurations, and generally far fewer. Questions about a particular stimulus could be repeated during the single trial during which it was presented (see below). As noted above, because additional exposure to stimuli increases the chance of success (DiPietro et al., 2002, Nagasaka et al., 2010), we intentionally limited the number of overall trials. Griffin received no more than 1-2 trials on any given day (again, see below); thus he could not engage in win/stay-lose/shift behavior (i.e., perseveration). 
Because all of Griffin's 3D experience with three- and four-cornered objects involved equilateral triangles and squares, we had to determine if Griffin was responding to the angles (local cues, $60^{\circ}$ and $90^{\circ}$ angles) rather than the entire figure (global cues) for the subjective contours. Thus, we additionally tested him on a right-angled triangle and a trapezoid, which would confound these possible angular preferences. We used one triangle and two different sizes of trapezoids, the latter to see if increasing the size would make him more likely to use a local rather than global cue.

\subsection{Modal and Amodal Stimuli Presentation}

Each of the 2D 2D stimuli was held vertically, approximately $15-20 \mathrm{~cm}$ from one of Griffin's eyes, to attract his attention (Fig. 6). The experimenter manually tracked the position of the stimulus with respect to the parrot's head to maintain presentation in front of one eye. Because no one has determined the extent of binocular overlap in a Grey parrot (either the angular overlap or, given the fairly large thick beak, the distance at which binocular overlap begins) nor the acuity of vision in the area of binocular overlap, we opted to constrain presentation to what was likely to be monocular observation (see Discussion). Griffin will not tolerate even a stray feather near his eye; thus using an eye patch to ensure monocular vision was not an option. The distances of the stimuli from Griffin's eye in this study were roughly the same as in our previous study on the Müller-Lyer illusion with the Grey parrot Alex (Pepperberg et al., 2008), in which we tested visual acuity at these distances. When Griffin made contact with the questioner with the other eye, he was asked "What shape X?" where X was the (non-black) color of the polygon in question; he provided a vocal English shape label ("\#corner", using possible labels \#=1,2,3,4,6,8).

[Insert Figure 6 about here]

\subsection{Formal Test Procedure}

Griffin's formal tests involved 38 modal and 33 amodal plus 5 detached probe trials. Most trials occurred on average 1-2 times/week from the end of May, 2010 to May, 2012, with breaks for experimenter absences, student intersessions, and some periods of noncompliance. 
Several additional trials occurred September-October 2012 after a medical absence of the first author, with a few trials given in March, 2014. All test questions were presented intermittently either during free periods (when Griffin was requesting various foods or interactions) or interleaved during sessions on current (and thus unrelated) experiments (e.g., training Griffin on bigger/smaller or testing him on exclusion concepts; Pepperberg et al., 2013).

Details of test procedures, including descriptions of precautions against inadvertent and expectation cuing are described in Pepperberg (1981, 1994); a summary is below. Only Griffin's first vocal responses that were distinct (as determined by the blind on-line evaluator) and shaperelated were scored (e.g., Pepperberg, 1981, 1987). For example, if he was inaudible, or repeated the color used in the query, his response was not counted; he was told "No", received a 1 sec time-out, and the question was repeated. We also repeated questions after an incorrect response in order to evaluate the source of his error, although we did not count his responses to repeated questions. For example, a few times he responded with the label for the shape of the black occluder instead of the designated target (defined by a non-black color). Here the examiner did not react except to wait a few seconds and then repeat the question to see if Griffin would repeat or alter his response. We reiterate that subsequent correct responses to error trials did not alter the "incorrect" score for that trial.

Presentation continued until an appropriate identification was made or four attempts occurred; errors were recorded. Occasionally, Griffin would engage in other forms of noncompliant behavior, by ignoring the questioner (e.g., by turning his back, engaging in extended preening, making requesting to return to his cage ["wanna go back"] or treats ["want nut"], or by consistently responding with labels clearly irrelevant to the current task (such as repeating the response to the immediately previous question, which would by design be on a different topic not requiring a shape response) or by refusing to answer at all; in such cases, the trial was dropped and repeated at a later date (see above, Pepperberg \& Gordon, 2005; Pepperberg \& Carey, 2102; Pepperberg et al., 2008). These situations were relatively rare. One 
trial was dropped and repeated for noncompliance in the amodal set (responding with irrelevant labels) and four in the modal set (one irrelevant label, three refusals to respond at all).

As in all studies with Griffin (and Alex before him), the protocol differed in two respects from ones used in other animal studies. These differences were also responsible for certain specific precautions to avoid inadvertent cuing. First, the task capitalized on Griffin's ability to work in the vocal mode. Rather than using an over-trained animal in an experiment with limited choices (2 alternatives; go, no-go), verbal responses give a much wider range of choices (conservatively, 1 of 6 possible shape labels in his repertoire; other vocal labels were also possible). As a consequence, Griffin was not making a peck to one of a small number of choices, so he could not be cued to look/peck in a particular direction. Second, each trial was presented intermittently during training and testing of other unrelated topics also under study. Griffin's responses thus had to be chosen from his entire repertoire (>30 vocalizations, including labels for foods and locations) and from among several possible experimental tasks using various exemplars and questions during each session. That is, although the presence of the specific stimuli likely did cue Griffin to the type of task being tested, he could not expect that all questions in a session would be related to shape and limit his responses on that basis; he still had to attend to the specific type of question being posed. For example, other tasks involved queries of "What color?", "What matter [material]?”, "What toy?", or "How many?” As noted above, only two trials, one for the modal data set and one for the amodal set, were repeated after noncompliant behavior (see above), and our choice of chance was not based on $>30$ possible responses.

Test situations included additional precautions to avoid cuing (note Premack, 1976, p. 132; Pepperberg, 1999). For all trials, we used a blind evaluator: A person other than the one presenting the tray (one of three possible individuals in these studies) vocally confirmed the answer (see Fig. 6 above). Only after this confirmation was Griffin rewarded (Pepperberg, 1981). Notably, in a previous study, we showed that the evaluator was not influenced by hearing the type of question posed. There, transcriptions of contextless tapes of another parrot's responses 
(Alex's) in a session agreed 98.2\% with original online evaluations (Pepperberg 1992); we believe the same would be true for Griffin.

Any direct cuing or training of Griffin as to the correct answer by the presenter was highly unlikely for at least three more reasons. First, because Griffin had not been trained on this task, and students who had trained shape labels were not involved in testing, no overlap occurred between the training and testing situations (Pepperberg, 1981); thus, for example, any possible facial tic or subtle gesture of the experimenter that cued a specific response would be unlikely to be identical in different humans. And, even if the experimenter did indeed mouth an initial sound and Griffin were somehow lip reading that human's face, one would expect that he would have confused "two" and "three" most often because of their initial phonemes, but, as seen in Results, those errors were never made. Second, because the specific response (one of several shape labels) varied from trial to trial, as did the stimuli (which were rarely repeated with respect to shape, and, as noted above, each stimulus was unique with respect to some aspect), Griffin could not be trained or cued as to one proper response to a particular stimulus. Finally, in a doubleblind test given previously given to Alex (Pepperberg \& Brezinsky, 1991; see also Pepperberg et al., 2008), in which the questioner was blind to the stimuli, which were hidden from her view in a box, efficacy rather than accuracy suffered: Alex was as correct as when the questioner could view the stimuli, but each trial took considerably longer as the experimenter could not focus the bird's attention on the task; we believe Griffin would respond similarly.

As in previous studies (e.g., Pepperberg, 2006), we videotaped a small percentage of sessions. Four such trials were digitized and the audio portions presented to three observers who had never interacted with Griffin. They were asked to repeat what they thought Griffin had said, to test for interobserver reliability with the on-line responders. If interobserver reliability with a blind, naive coder was high, we could be assured of the validity of other trials. All three people agreed 100\% with each other and with the original blind observers on Griffin's responses on all four trials. Replicates of two of the video trials are included as supplementary material (the 
listener/evaluator filmed the replicate trials to ensure clarity for publication and thus was not acting as an evaluator). Given that Griffin was more interested in the student performing the taping and the video camera rather than the objects to be labeled, such trials were difficult to execute and kept to a minimum.

\section{Results}

Despite the lack of training on 2D items and limited exposure to the amodal, modal, and detached probe stimuli, Griffin immediately transferred from 3D stimuli and responded appropriately to the test figures. Griffin's accuracy was high, at 76\% (29/38 trials) for modal completion and $70 \%$ (23/33 trials) for amodal completion trials; he was also $100 \%$ correct on all five detached probe trials in which no occlusion occurred. Taking chance at $20 \%$ (because, as noted above, we excluded the 8-cornered shape in both studies), this performance was statistically significant (on a binomial test, $p<<0.01$, for all types of trials, including detached probes). Even if we choose to use chance of 0.5 on the amodal task (one might argue that he should respond either with the label of the occluded shape or give the number of visible corners of the actual object), his data are still statistically significant $(p<0.01)$. As noted in the sections describing the details of his responses, he made no errors on squares and hexagons, and few on regular 2-cornered polygons; the majority of errors occurred for 1- and 3-cornered polygons and triangles, which he seemed to confuse in both modal and amodal tasks. Figure 7 presents the overall summary of Griffin's responses.

[Insert Figure 7 about here]

To best appreciate in detail Griffin's success on these tasks, we next present the data in a confusion matrix form for the amodal and modal experiments separately. In each matrix the columns represent the actual "shapes" presented to Griffin (1-, 2-, 3-, 4- and 6-cornered objects) and rows represent how he labeled them; that is, his vocal responses (“\#-corner”). The numbers within each shape denote the number of times a variant of that shape was presented. Diagonal entries 
represent correct responses, off-diagonal entries represent errors.

\subsection{Amodal Completion}

As noted above and considering that chance was $20 \%$, Griffin's accuracy with amodally completed stimuli was very high $(23 / 33$; Fig. 8) showing that he responded to the true shape of figures, despite the fact that all were partially hidden. In detached probes (such as those shown in Figs. 5d, h), where no occlusion occurred, he responded to figures never before seen (irregular polygons) with the number of visible corners. He was correct on the very first trial of the experiment, which suggested he immediately transferred his response from a non-occluded 3D item to drawings of occluded polygons. He did as well at the beginning of tests as at the end; a Fisher exact test comparing his scores on the first versus the last fifteen trials produced a $p=0.68$, supporting the view that he did not learn the task over time. His errors, although relatively few, are of interest. Some seem to be due to inattention (labeling the shape of the occluder), others suggest possible errors in processing (e.g., conflating 1- and 3-cornered objects). We raise the possibility of such interpretations in the Discussion. Note that in the majority of cases, when he was asked a second time about figures on which he erred, he provided a correct response without any additional input from the examiner. However, he was never scored "correct" on such trials.

\section{[Insert Figure 8 about here]}

\subsection{Modal Completion (subjective contours)}

For modal test stimuli, in contrast to amodally completed stimuli, it is important to emphasize that there is nothing in common at an image level between the trained and test stimuli. In the amodal case, the trained 3D and tested 2D images do have some local features in common (a 60 or 90 degree corner); in the modal completion case the trained 3D and tested 2D images share nothing at an image level. As such, the modal task provides the strongest case for an invariant representation of shape, and, notably, Griffin was as good on modal stimuli (29/38 correct trials) as amodal stimuli. He again was correct on his very first trial, showing he could recognize these 2D stimuli without any training. 
And, again, no significant difference existed between his scores on the first versus the last fifteen trials, a Fisher's exact test yielding a $p=0.22$, indicating a lack of learning during these trials. Almost all errors appeared to involve confusion between 1- and 3-cornered items. We again note various possible interpretations for his behavior in the Discussion. Notably, Griffin was not quantifying pacmen or numbers of circles, because in no instance did an error correspond to the quantity of these objects. In the majority of cases, when queried a second time on figures on which he erred, he responded with the correct label. As before, he was never scored "correct" on such repeated trials.

[Insert Figure 9 about here]

\section{Discussion}

Our basic finding is that Griffin, trained for approximately 15 years with real 3D shapes, was remarkably adept at identifying shapes in tests on 2D drawings. With essentially no training on any pictorial forms, he vocally labeled 2D partially occluded shapes (amodal completion) and figures bounded by subjective contours (modal completion). The data not only demonstrate his capacity to interpret a 2D image, but also his ability to visually "infer" the true shape of an even partially visible object or, for subjective contour stimuli, when nothing of the trained image remained. His performance, if not quite equal to that of humans in similar tasks, was extremely good considering his unfamiliarity with any of the testing stimuli and the large differences between trained and tested images.

Against this remarkable success, a substantial literature exists showing that, with a very few exceptions (see below), animals either do not show these completion phenomena at all or show some degree of success only after having undergone considerable training with very closely related stimuli. Even a study such as that of Tvardíková and Fuchs (2010), which involved the reactions of wild birds (various species of Paridae) to occluded versus amputated models of a predator (Accipiter nisus) attached to a feeder, provides only circumstantial evidence for amodal completion. Unfortunately, we cannot rule out a simpler explanation based on the likely previous experience of the tits where the fully visible hawk could at the next moment become partially occluded (or the 
reverse), thus allowing an associative connection between the occluded image and the full image of fearsome hawk. As such, this specific pairing and not more general process of amodal completion could explain the results. Thus, although both types of completion have been reported in various nonhuman species, indicating that the capacity could rely on relatively early-level processing and possibly a single mechanism that is widespread across taxa (Ben-Yosef \& BenShahar, 2012; Kalar et al., 2010; Nakayama et al., 1995), those results are not fully convincing. In some instances where training was not an issue, success or failure may have involved mechanisms unrelated to the perceptual ones being examined here, and performance may have actually relied on mechanisms that do not match those of humans (e.g., luminance, aspect ratio; Minini \& Jeffery, 2006). In other instances, actual tasks differed considerably among the laboratories (with respect to, e.g., motion, 2D vs 3D stimuli, CRT vs LCD monitors [i.e., flicker fusion effects] and pre-exposure to stimuli; Nagasaka, Lazareva, \& Wasserman, 2007; Nagasaka \& Wasserman, 2008; Vallortigara, 2006) with the consequence that results also varied considerably (e.g., Aust \& Huber, 2006; DiPietro et al., 2002; Lazareva Wasserman, \& Biederman, 2007; Sekuler, Lee, \& Shettleworth, 1996; Ushitani \& Fujita, 2005; Ushitani, Fujita, \& Yamanaka, 2001).

For these reasons, we argue that Griffin's completion results are singular. What allows us to make this claim when numerous efforts in the past have not been as successful? Many reasons are possible, as several significant differences exist between our study and previous attempts. The most striking were differences in the intensity and generality in learning about real 3D objects. Unlike most (but not all; see the Nagasaka et al., 2010 study on bonobos) nonhumans tested for modal and amodal completion is the richness of Griffin's visual experience outside of test procedures. Human children need such experience in order to develop these abilities (e.g, Kellman \& Spelke, 1983), and from the time of his entrance into the laboratory at $7.5 \mathrm{wks}$, Griffin's 16 years of daily life had been more like that of a human toddler than an experimental laboratory subject. Thus he likely saw items in the laboratory from their 3D "physical" perspective but also, at times, from perspectives that would seem more 2D (e.g., the flat surface of a data sheet that was then handled in a 3D manner by a human). 
Such experiences may have assisted in his transfer from 3D to 2D representations (note Stephan et al., 2013). Moreover, although trainers and testers were careful not to occlude 3D objects used as initial exemplars in this study, many other objects in the lab, including food items, would be seen by Griffin as being partially occluded while being handled by humans in his presence. Griffin watched as caretakers held and chopped fruits and vegetables, as they removed objects from his cage and environment for cleaning, as they handled other exemplars in training and their own possessions e.g., picked up books, laptops, articles of clothing. Thus, he was provided with two well known advantages to foster deep and invariant learning: spaced practice and varied exemplars (e.g., Billing, 2007; Karpicke \& Bauernschmidt, 2011). As a consequence, his ability to recognize 3D occluded objects as their non-occluded counterparts would not be surprising and, possibly, aided his ability to transfer to both 2D amodal representations and modal completion tasks.

Contrast these experiences to most animal studies in which the very limited number of shapes to be learned were generally 2D figures on a $2 \mathrm{D}$ background, and where testing conditions closely resembled those of training. Furthermore, both amodal and modal completion are inherently three dimensional processes, requiring an implicit understanding of the $2 \mathrm{D}$ stimuli as representing $3 \mathrm{D}$ forms, as depicted in Figure 10. Seeing only 2D projections of an object would not likely enable an animal to develop or generalize to an invariant 3D representation of that object. Notably, baboons failed when tested for amodal completion in a task involving considerable training on 2D figures and forced, two-choice testing on 2D transfer stimuli (Deruelle, Barbet, Dépy, \& Fagot, 2001) but did succeed (although only after several hundred training trials and 60 forced, two-choice testing trials) when given both training and testing stimuli that provided background depth cues indicating that the occluder was indeed in front of the targeted object (Fagot, Barbet, Parron, \& Deruelle, 2006; see also Nagasaka et al., 2010 on bonobos). Interestingly, an amodal completion experiment with the chimpanzee Ai (Sato, Kanazawa, \& Fujita, 1997), involved motion of rods behind occluders, which also provided some level of depth cues (however, other, non-amodal completion explanations of their data are also likely; see below.) 


\section{[Insert Figure 10 about here]}

Note, too, that Griffin understood symbolic representation: For over 16 years, he had been taught, as depicted in Figure 3, in both a comprehension and production mode, that vocal labels represented colors, shapes, materials, foods, locations, category labels, and to a limited extent, that single Arabic numerals could represent sets of multiple objects (e.g., Pepperberg \& Carey, 2012). Given this equivalence, he would be more likely than most other nonhuman subjects to understand that a $2 \mathrm{D}$ form could be a representation of a 3D object. Notably, bonobos in the amodal completion study of Nagasaka et al. (2010) were also adept at labeling and thus symbolic representation.

We do not claim that advanced cognitive abilities are necessarily needed for the visual processes described here (see Nakayama et al., 1995, Nieder, 2002). What we believe to be special about Griffin is his ability to encode vocally what he has seen so as to respond in a manner similar to that of humans. This symbolic encoding ability likely does require advanced cognitive capacity. Notably, parrots (like corvids) have specific brain areas known not only to be responsible for cognitive processing but that are also, relative to overall brain size, comparable to those of nonhuman primates (Iwaniak, Dean, \& Nelson, 2005: see also Jarvis et al., 2005). Grey parrots have shown advanced cognitive processing in other domains that are equivalent to or even more advanced than those of nonhuman primates (e.g., Pepperberg, 1999; Pepperberg \& Hartsfield, 2014) and equivalent to young children (Pepperberg \& Carey, 2012). Griffin's abilities thus should not be particularly surprising.

We further compare our study with those using other subjects and examine possible competing explanations for our results. We first investigate whether Griffin responded preferentially to local features of an image rather than having a robust response to the actual shapes, which were only partially visible. Next, we examine if Griffin simply counted corners; that is, if he had no underlying representation of shape. We then explore the issue of stimulus generalization, commonly raised in many animal studies; here concern exists that training and testing stimuli are sufficiently close such that subjects base responses purely on similarity. We also contrast our work with studies 
in which a subject might base responses on similarities in mass and/or number of items between training and test stimuli and, more broadly, we discuss the differences between a subject that can be tested using English speech versus those that must be tested based on variations of match-to-sample.

\subsection{Griffin's success in relation previous studies}

Results in some studies may be attributed to the use of local cues. Although mice responded in a manner consistent with experiencing amodal completion, the researchers involved (Kanizsa et al., 1993) were careful to note that alternative explanations could not be ruled out, such as similarities in testing and training stimuli based on local cues. Honeybee success in the study by Van Hateren et al. (1990) on illusory contours can, for example, be explained by local cues, and the authors themselves are very careful to state that their results are not compelling with respect to claims that their subjects actually see the illusory contours as do humans. Pigeons' failures on completion studies have been attributed to a preference for responding to local rather than global features (e.g., Fujita et al., 2012; Prior \& Güntürkün, 1999).

We thus examine Griffin's responses. Could the small number of mistakes he made be predicted from a 2D local cue interpretation suggested by Fujita? We claim that such cues, although possibly responsible for his occasional confounds of 1- and 3-cornered objects (a minority of 76 total trials), were different from the type of local attentional processing used by pigeons.

During amodal completion, Griffin did not label 1- and 3-cornered items as 2- or 4-corner, respectively (see Fig. 51, 5c respectively), which would have indicated no understanding of occlusion. Rather than focusing on local intersections of occluder and targeted form, he likely quickly scanned the entire stimulus, focusing on a different local cue without carefully checking numbers of angles. For example, stimuli in cells $(1,3)$ and $(3,1)$ in Figure 8 might look the same to a subject that is not carefully attending to the non-occluded part. As noted above, in three of these four cases, when asked a second time, Griffin responded correctly. Such self-correction cannot prove that the original response was caused by inattention; however, Griffin showed he knew when to switch focus, and thus when attention to a local cue was inappropriate. He was thus unlike the pigeon subjects. 
Similarly, the majority of Griffin's modal completion errors (6/9) also involved conflating 1and 3-cornered items, possibly by focusing on a single angle/point - the $60^{\circ}$ apex [see cells $(1,3)$ and $(3,1)$, Fig. 9], characteristic of triangles as well as objects with fewer corners. Here, however, his errors raise a different possibly important issue: He possibly identified his regular polygonal training items by their angles and conceivably transferred this knowledge to modal completion tasks. For example, the $60^{\circ}$ angle of the trained triangle matched that of the mouth of the pacman for most subjective triangles, and could have cued his response; likewise the $90^{\circ}$ angle (square) and the $120^{\circ}$ angle (hexagon). Such was not an issue in amodal completion, because he succeeded on probes. We addressed this issue in modal completion by additional test trials [see cells $(3,3)$ and $(4,4)$ in Fig. 9] where a single stimulus contained non-standard polygonal angles. Given a right-angled triangle stimulus, Griffin correctly labeled it 3-corner despite the $90^{\circ}$ angle. Given a small version of a trapezoid (bases of $3.5 \mathrm{~cm}, 7 \mathrm{~cm}$ ), he appropriately labeled it 4-corner. Had he responded only to the size of the angles, he would have replied 3- or 6-corner. Thus he was not examining only the angles. However, given a much larger trapezoidal figure (bases of $5.5 \mathrm{~cm}, 11 \mathrm{~cm}$; cell $(3,4)$ in Figure 9) where focusing on the entire object would be more difficult, he first called it 3-corner, apparently attending to a lower angle. Notably, when asked a second time, he responded appropriately (as he did for other errors on modal completion). Again, such self-correction does not prove, but does suggest, that the original errors likely involved inattention and an ability to refocus.

Griffin's data also differed from those of primates in amodal completion. For example, Fujita and Giersch (2005) trained capuchin monkeys to match monochrome broken rods to broken rods, intact rods to intact rods, and two types of mid-line occluded rods to their respective matches, then tested them with sets of the same monochrome rods with their monochrome occluders, but now with additional, differently colored occluders placed in three positions: roughly one-third along the length of the rod, midway (covering the monochrome occluder), and roughly two-thirds along the length of the bar. The authors claim that the midway occlusion was the critical test, and that because their monkeys matched that occlusion to the single intact rod, they understood amodal completion. 
However, monkeys' responses could simply have been made based on exclusion and local cues: If the break or monochrome colored occluders were not visible (and they were visible on the one-third and two-third versions of occlusion), then the stimulus would likely be categorized (according to prior training) as the intact rod. Other experiments in that series could also be explained in a similar manner.

\subsection{Counting Corners?}

Our parrots learn to identify polygonal shapes according to their number of corners, that is, to state "\#-corner" when asked "What shape?" (Pepperberg, 1987b); they are also trained on number (“How many?") and shape questions simultaneously. This procedure is a matter of linguistic convenience and symbolic consistency for ourselves and perhaps for the parrots. Unanswered therefore is whether each figure was apprehended as a pictorial shape or coded by its specific number of corners. Until now, we could not distinguish between these two alternatives.

Given such training, we might have expected Griffin to respond with the number of visible corners rather than to the occluded shape for amodal completion, and possibly to the number of corners or number of pacmen for Kanizsa figures. Such was not the case. In amodal completions, he responded as though he understood we were referring to the non-occluded shape, except as expected in detached probes (e.g., Fig. 5d, 5h), where he responded to non-regular polygons (figures never before seen), with the actual number of visible corners. In those probes, counting corners was, of course, the only possible correct response, whereas in the occluded figures he had a choice and he chose to respond as would humans. For modal completion, he was clearly not labeling the total number of corners or pacmen. When, for example, he erred once on a 2-cornered Kanizsa figure with an additional pacman, he labeled it 1-corner. Similarly, were he labeling pacmen, he could have, for example, labeled one of the 6-cornered stimuli as 8-corner. Furthermore, as noted above, his conflation of 1- and 3-cornered objects in both amodal and modal trials suggests he could be more sensitive to the general shape than to the actual number of corners. Thus, again, although Griffin made occasional errors, he responded appropriately at a statistically significant level, indicating a 
robust understanding of shape.

\subsection{Visual Experience and Stimulus Generalization}

Taught to identify 3D objects, Griffin transferred without training to 2D amodal stimuli; other avian subjects such as pigeons seem to need extensive experience with 2D stimuli for amodal completion (Nagasaka et al., 2007). Griffin did need considerable training to learn to identify 3D shapes with English vocal labels; however, the length of that training likely was a consequence of the difficulty in learning to configure his vocal tract in specific ways to produce English speech patterns rather than problems with recognition of specific objects (see Patterson \& Pepperberg, 1994, 1998; Pepperberg \& Carey, 2012). Thus, unlike other nonhumans, who are generally trained on 2D stimuli quite similar to those on which they will be tested (review in Fujita et al., 2012), essentially all of his training occurred with 3D stimuli: wooden, plastic or Playdoh objects of $\sim 3-5 \mathrm{~cm}$ per side and 0.5 $0.7 \mathrm{~cm}$ thickness, or some woolen or paper cut-outs of $\sim 3-4 \mathrm{~cm}$ per side that he could still chew and interact with as 3D objects. In contrast, all test stimuli were then $2 \mathrm{D}$ items, printed on paper he was not allowed to chew, with irregularly shaped backgrounds such that they were $\sim 10 \mathrm{~cm}$ x $15 \mathrm{~cm}$. He had never seen either the occluded 2D objects nor the nonregular polygons used in amodal completion tests. That is, he was not simply deciding if the target was more or less similar to another figure (e.g., Nagasaka et al., 2007, Regolin et al., 2004), but rather what exactly to label each stimulus. His ability to label some of the regular-polygon shaped occluders occasionally interfered with the targeted task, but not to any significant extent.

As mentioned before, animals showing the perception of subjective contours and figures (modal completion) is of even greater importance than that of recognizing partially occluded figures. At the image level in this subjective contour case, there is no overlap between training and test stimuli and this represents the most extreme test of shape invariance. In reviewing studies claiming to show modal completion in nonhumans (e.g., chicks responding correctly to subjective contours, Zanforlin, 1981; for pigeons, see Prior \& Güntürkün, 1999), these can be more simply understood as more conventional associative learning and stimulus generalization. According to Horridge et al. 
(1992), the bees in their experiments, tested on similarly constructed but somewhat differently oriented stimuli, also did not perform particularly well in terms of illusory contours. Griffin's data with shapes defined by subjective contours argues even more strongly than do his data on occlusion against an explanation based on stimulus generalization. Such an explanation relies on some portion of the testing stimulus resembling that of the training stimulus. He had, however, never been exposed to pacmen prior to this study, and could not label them nor the black circles initially used as occluders. Overall, the difference between training and testing stimuli are so great and the accuracy of his identifications so high, that stimulus generalization can be ruled out.

Another reason to exclude stimulus generalization for both tasks lies in the small number of trials needed to conduct our study. Unlike other nonhuman subjects, Griffin's results did not depend on statistical averaging of hundreds of trials (e.g., Deruelle et al., 2000; Di Pietro et al., 2002) involving pecking behavior to a limited set of choices (e.g., for amodal completion, between a whole and very closely-related partial figure), but rather on vocal English responses, one of a subset of 6 chosen from an entire repertoire of roughly 30 labels. Notably, the hundreds of test trials in other nonhuman studies provide the possible opportunity for new learning. In contrast, Griffin had fewer than 40 test trials each in the amodal and modal completion tasks, and fewer probes (although equal on a percentage basis), much as would be done with humans (e.g., Bulf, Valenza, \& Simion, 2009). Furthermore, although the basic stimuli types for both modal and amodal trials were repeated several times, we used few repetitions of the same stimulus class (see Figs. 8, 9) compared to other studies, and all stimuli were unique in some manner; that is, differed with respect to size of the target and/or occluding figures or pacmen, distances between pacmen, or color. Moreover, as noted above, Griffin did not need experience with oddly shaped items in order to label the detached probe stimuli. Interestingly, he seemed to recognize never-before seen free-standing figures in such trials as atypical - that is, non-canonical - and quantified corners only for these. Finally, again as noted earlier, probes also demonstrated that Griffin had not somehow learned to quantify pacmen for the Kanizsa figures; in one case, for example, he could simply have stated the largest number he knew 
(“8”), but did not.

\subsection{Mass and Numerical-Based Responses}

Finally, Griffin's responses to amodal stimuli were not based on number or mass of items: For example, in some tests, nonhuman subjects, after being trained on, for example, one object (a polygon) then were asked to choose between one occluded polygon and two fragments (the polygon minus the occluder and the occluder nearby; e.g., Regolin \& Vallortigara, 1995); thus responses could be made with respect to the number of items displayed and not on completeness. Results of Sato, Kanazawa, and Fujita (1997) for the chimpanzee Ai (well-known for her work on labeling and number concepts; see Matsuzawa, 1995; Mirofushi, 1997), although consistent with amodal completion, might also be consistent with the ape initially responding based on number: Ai was trained to match intact rods to intact rods and broken rods to broken rods, then tested with occluded rods such that the top and bottom portions moved synchronously, asynchronously, or only one part moved; all test responses were rewarded. She may have responded based on the number of different motions observed, matching number of motions to number of rods, receiving many rewards. In subsequent tests, each using over 100 repeated initial training trials, she may have responded based on the similarity of the test stimulus to the reward pattern she experienced in the first set of tests. In other tests (e.g., Nagasaka et al., 2007, 2010), nonhuman subjects were trained to an occluded object and then their errors to the non-occluded object or to the object missing a part were examined; errors to the non-occluded object — that having a closer mass to the occluded object—were considered evidence of amodal completion. Again, Griffin simply labeled the stimulus that he viewed.

\subsection{Attention and Laterality}

Regolin et al. (2004), after studying amodal completion in chicks whose right or left eyes had been temporarily patched, showed that the information garnered by the left eye allowed the chick to

process information about the entire figure, whereas those receiving information from the right eye focused on local features. As noted above, Griffin primarily processed information about entire 
figures. Might his success be due to a viewing side preference?

Given that extent of binocular overlap in Grey parrots is unknown, and can be estimated only from data on Senegal parrots ( $30 \%$, according to Demery et al., 2011$)$, a not very closely related species, we chose to present stimuli monocularly. Interestingly, Griffin tended to shift his head so as to view stimuli with his left eye as they came into view; he did, however, potentially have a quick, initial binocular view. If parrot vision more closely resembles that of chicks than pigeons (Harrison, pers. comm.), then his propensity to use the left eye suggests he could perceive the figures globally, rather than locally (see Regolin et al., 2004), unlike pigeons (review in Güntürkün, 1997, but see Goto et al., 2004). The majority of Griffin's errors, for both modal and amodal stimuli, were confounds of 1- and 3-cornered objects, which, as we discuss above, seem related to attention, that is, of choosing to focus quickly on one segment of the stimuli rather than on the whole, possibly in order to move on to more interesting tasks in the laboratory (NB: Vallortigara et al., 2008, discusses how different situations can call forth different processing abilities). Griffin received no punishment other than a lack of a favored treat, so that the cost of an error was minimal, and he confused the 1- and 3cornered items less often overall than he labeled them correctly. Possibly, parrots' frontal vision is somewhat myopic for focusing on foraging local details (see, e.g., Fernández-Juricic, 2012; Prior \& Wilzeck, 2008) and Griffin's attention for the $60^{\circ}$ apex might have been caught as the stimulus was brought into view binocularly. Interestingly, during initial 3D training in 2009, his most often errors on both 1- and 3-cornered objects was to label them "2-corner", suggesting that, at the start of training, he lumped all objects with a $60^{\circ}$ apex into a single category. Here such errors were exceedingly rare (one error on a modal completion). Again, overall, his errors do not detract from the finding that he was not confused by having an object occluded or by it being illusory.

\section{Conclusion}

At least one Grey parrot, Griffin, has been shown to have a sophisticated concept of shape. He transferred from training on 3D stimuli to testing on 2D stimuli. He could perceive and label occluded and Kanizsa figures, that is, demonstrate both amodal and modal completion. Many other 
creatures must experience this problem in their daily lives (e.g., Lea, Slater, \& Ryan, 1996; Regolin \& Vallortigara, 1995). For example, processing partial clues about a potential predator and reacting is safer than not, even if some false alarms incur costs. Thus we expect that many nonhumans are able to detect something about amodal and Kanizsa figures. However, Griffin performed in a manner more sophisticated than other laboratory-based subjects. His testing stimuli differed considerably from his training stimuli, and his test trials were interspersed with tests on several other topics; he had to choose his responses from among approximately 30 in his overall vocal repertoire and, even if we grade him conservatively, among six possible labels for shapes. He could distinguish between occluded regular (canonical) polygons and non-occluded non-canonical ones, labeling the number of visible corners only on the latter stimuli. Thus he, unlike most subjects in such studies, was not 'taught to the test" and, by labeling exactly what it was that he had perceived, demonstrated that he could generalize his concept formation in a particularly robust manner.

\section{A challenge to future object recognition research in brains and machines.}

We have shown that, under the conditions of our study, Griffin's capacities to recognize occluded figures and shapes for which key outline contours are missing (subjective contours) compare favorably with those of human observers. Data for humans originate with the pioneering studies of perceptual psychologists such as Kanizsa (1979) and were more fully elaborated in recent work (e.g., Bregman, 1994; Nakayama et al., 1995). The current study and all those studies of human observers indicate that object recognition can occur readily for specific images that, pixel-wise, have not been experienced. We believe that the work presents two challenges to examine the limits and extent of such abilities: one challenge for those studying nonhumans and another for researchers interested in the neurophysiological and computational basis of object recognition.

In our review of studies on nonhuman capacities for modal and amodal completion, an important factor appears to be long training or at least experience with 3D stimuli. No studies have, however, directly compared the capacities of nonhumans with and without such experience on these tasks. We note, for example, that homing pigeons, living as a group in an outdoor aviary, and given 
experience with a number of 3D items, could correctly classify novel 2D photos of these objects whereas birds without such 3D experience failed (Stephan et al., 2013). How might the two different groups fare on studies of modal and modal completion? Macaque monkeys can learn to discriminate shapes which differ very little from each other and recent studies (Livingstone et al., 2014) have shown that multiple response mappings (up to 20 alternatives) can be obtained. Might the same processes we see in the parrot be found in monkeys with extensive discriminative experience?

We also argue that current results constitute a challenge to contemporary models, computational (Krizhevsky, Sutskever, \& Hinton, 2012; LeCun \& Bengio, 1995) and neurophysiological, of object recognition (Hung, Kreiman, Poggio, \& DiCarlo, 2005; Maja, Hong, \& Solomon, 2015). Although these models have a very impressive record with respect to discriminating a wide range of novel (never before seen) shapes, they have not been tested when new objects, particularly untextured ones, have been occluded, the case of amodal completion described here. More importantly, they have not been tested on shapes defined by subjective contours, an even more stringent test because no shared image features exist. A neurophysiological study would not be easily feasible, but machine vision experiments to simulate Griffin's learning and testing are. A number of variants would need to be explored. Would the network learn without any specific examples of polygons being occluded? Could it learn occlusion via training with other objects? Could it learn without experiencing any occlusion at all? At stake here is whether a neural network, dealing only with raw images, without any explicit instruction, can perceive information about surfaces (Nakayama \& Shimojo, 1992; Nakayama et al., 1995). Such network capacity has never been explored and could constitute a significant achievement and extend the domain over which such algorithms apply. Success could in turn raise issues as to how information about surfaces is learned in biological systems. Alternatively, failure of such networks under a wide variety of conditions would raises alternative issues, leading the inquiry as to how such mechanisms are either preprogrammed or otherwise learned in biological systems. 
Acknowledgements This research was supported in part by a National Science Foundation grant BCS-0920878 (Nakayama, PI; Pepperberg, co-PI ) and donors to The Alex Foundation (particularly the Sterner Family, The California Community Foundation, Anita Keefe, the Marc Haas Foundation, Janice Boyd, Joseph Golden, Alex and Michael Shuman, Nancy Chambers, Kathryn and Walter McAdams, Megumi Oka and the Makioka Foundation, Nancy Sondow, Katie Dolan, Deborah Rivel Goodale/Wildtones, The Robert D. Goodale Fund (via the Indian River Community Foundation), Elva and Bob Mathieson, Jay Sugarman, Debby and Michael Smith, Rose and Ken Lawlor, George and Lisa Bono/The Platinum Parrot, Regina and Ed Underwood, John Paton, Ann Arbor Cage Bird Club, Arlene Levin Rowe/Grey Parrot Studios, Daisy Paradis/The Howard Bayne Fund, Dan Vollmer, the Dutch Parrot Foundation (Stichting Papegaai), Phoenix Landing, The South Bay Bird Society, Mark Hagen, the Canadian Parrot Society, Debbie Rijnders/Tinley Advies \& Producties BV, Vanessa Rolfe, Leigh Ann and Carl Hartsfield, Jean Berko Gleason, Mary Ogg, Featherlust Farms Bird Shop, James Armstrong, Reno Area Avian Enthusiasts, Janet Trumbule, Don and Grace Wheeler, Pat Hill, Roni Duke, the Medwick Foundation, Tammy Carreiro, Carol Samuelson, Nancy Clark and Bill Broach, Rocky Mountain Society of Aviculture, Greg LaMorte, the Andrew De Mar Family, Foundation, Ann Dey, Tracy Hylka, Charlene Bebko, Ellen Paul, Roxanne and Scott Bok, Hunt Associates, Jan and Jeff Strong, and Mary Long). We thank Harrison's Bird Food, the Lafeber Company, and Gini's Birdie Bread for food and treats. The study procedures comply with the current laws of the country under which they were performed (U.S.A.). 


\section{References}

Aust, U., \& Huber, L., (2006). Does the use of natural stimuli facilitate amodal completion in pigeons? Perception, 35, 333-349.

Bakin, J.S., Nakayama, K., \& Gilbert, C.D. (2000). Visual responses in monkey areas V1 and V2 to three-dimensional surface configurations. The Journal of Neuroscience, 20(21), 81888198.

Bandura, A. (1971). Analysis of modeling processes. In A Bandura (Ed.), Psychological modeling (pp 1-62). Chicago: Aldine-Atherton.

Ben-Yosef, G., \& Ben-Shahar, O. (2012). A tangent bundle theory for visual curve completion. IEEE Transactions on Pattern Analysis and Machine Intelligence, 34, 1263-1280.

Billing, D. (2007). Teaching for transfer of core/key skills in higher education: Cognitive skills. Higher Education, 53, 483-516.

Bowmaker, J.K., Heath. L.A., Das, D., \& Hunt, D.M. (1994). Spectral sensitivity and opsin structure of avian rod and cone visual pigments. Investigative Ophthalmology and Visual Science, 35, 1708.

Bowmaker, J.K., Heath, L.A., Wilkie, S.E., Das, D., \& Hunt, D.M. (1996). Middle-wave cone and rod visual pigments in birds: Spectral sensitivity and opsin structure. Investigative Ophthalmology and Visual Science, 37, S804.

Bregman, A. S. (1994). Auditory scene analysis: The perceptual organization of sound. Cambridge, MA: MIT Press.

Bulf. H., Valenza, E., \& Simion, F. (2009). The visual search of an illusory figure: A comparison between 6-month old infants and adults. Perception, 38, 1313-1327.

Carvalho, L.S., Knott, B., Berg, M.L., Bennett, A.T.D., \& Hunt, D.M. (2011). Ultraviolet-sensitive vision in long-lived birds. Proceedings of the Royal Society B, 278, 107-114.

Conci, M., Müller, H.J., \& Elliott, M.A. (2007). The contrasting impact of global and local object attributes on Kanizsa figure detection. Perception \& Psychophysics, 69, 1278-1294.

Cuthill, I.C., Hart, N.S., Partridge, J.C., Bennett, A.T.D., Hunt, S., \& Church, S.C. (2000). Avian color vision and avian color playback experiments. Acta Ethologica 3, 29-37.

Demery, Z.P., Chappell, J., \& Martin, G.R. (2011). Vision, touch and object manipulation in Senegal parrots, Poicephalus senegalus. Proceedings of the Royal Society B, 278, 3687-3693.

Deruelle, C., Barbet. I., Depy, D., \& Fagot, J. (2000). Perception of partly occluded figures by 
baboons (Papio papio). Perception, 291, 483-1497.

DiPietro, N.T., Wasserman, E.A., \& Young, M.E. (2002). Effects of occlusion on pigeons' visual object recognition. Perception, 31, 1299-1312.

Fagot, J., Barbet, I., Parron, C., \& Deruelle, C. (2006). Amodal completion by baboons (Papio papio): contribution of background depth cues. Primates, 47, 145-150.

Fernández-Juricic, E. (2012) Sensory basis of vigilance behavior in birds: Synthesis and future prospects. Behavioural Processes, 89, 143- 152.

Fujita, K., \& Giersch, A. (2005). What perceptual rules do capuchin monkeys (Cebus paella) follow in completing partly occluded figures? Journal of Experimental Psychology: Animal Behavior Processes, 31, 387-398.

Fujita, K., Nakamura, J., Sakai, A., Watanabe, S., \& Ushitani, T. (2012) Amodal completion and illusory perception in birds and primates. In O.F. Lazareva, T. Shimizu, \& E.A. Wasserman (Eds.), How animals see the world (pp. 101-116). Oxford: Oxford University Press.

Gajdon, G.K., Amann, L., \& Huber, L. (2011). Keas rely on social information in a tool use task but abandon it in favor of overt exploration. Interaction Studies, 12, 304-323.

Gobert, J.D., Baker, R., \& Sao Pedro, M. (2011). Using machine-learned detectors to assess and predict students' inquiry performance. Paper presented at the Society on Research for Educational Effectiveness, September.

Goldsmith, T., \& Butler, B.K. (2005). Color vision of the budgerigar (Melopsittacus undulates): hue matches, tetrachromacy, and intensity discrimination. Journal of Comparative Physiology A, Neuroethology, Sensory, Neural, and Behavioral Physiology, 191, 933-951.

Goto, K., Wills, A.J., \& Lea, S.E.G. (2004). Global feature classification can be acquired more rapidly than local-feature classification in both humans and pigeons. Animal Cognition, 7, $109-113$.

Horridge, G.A., Zhang, S.W., \& O’Carroll, D. (1992). Insect perception of illusory contours. Philosophical Transactions of the Royal Society London B, 337, 59-64.

Hung, C. P., Kreiman, G., Poggio, T., \& DiCarlo, J. J. (2005). Fast readout of object identity from macaque inferior temporal cortex. Science, 310, 863-866.

Iwaniuk, A.N., Dean, K.M., \& Nelson, J.E. (2005). Interspecific allometry of the brain and brain regions in parrots (Psittaciformes): Comparisons with other birds and primates. Brain, Behavior, \& Evolution, 66, 40-59. 
Izawa, I-E., \& Watanabe, S. (2011). Observational learning in the large-billed crow (Corvus macrorhynchos): effect of demonstrator-observer dominance relationship. Interaction Studies, 12, 281-303.

Jarvis, E.D., Güntürkün, O., Bruce, L., Csillag, A., Karten, H., Kuenzel, W., et al. (2005). Avian brains and a new understanding of vertebrate brain evolution. Nature Reviews Neuroscience, $6,151-159$.

Kalar, J., Garrigan, P., Wickens, T.D., Hilger, J.D., \& Kellman, P.J. (2010) A unified model of illusory and occluded contour interpolation. Vision Research, 50, 284-299.

Kanizsa, G. (1955/1987). Margini quasi-percettivi in campi con stimulazione omogenea. Rivista di Psicologia, 49, 7-30 [translated in S. Petry \& G. Meyer (Eds,), 1987 The Perception of Illusory Contours. New York: Springer]

Kanizsa, G. (1979). Organization in vision: Essays on gestalt perception. Santa Barbara, CA: Praeger Publishers.

Kanizsa, G., Renzi. P., Conte, S., Compostela, C., \& Guerani, L. (1993). Amodal completion in mouse vision. Perception, 22, 713-721.

Karpicke, J.D., \& Bauernschmidt, A. (2011). Spaced retrieval: Absolute spacing enhances learning regardless of relative spacing. Journal of Experimental Psychology: Learning, Memory, \& Cognition, 37, 1250-1257.

Kellman, P.J., \& Spelke, E.S. (1983). Perception of partially occluded objects in infancy. Cognitive Psychology, 15, 483-524.

Krizhevsky, A., Sutskever, I., \& Hinton, G.E. (2012). Imagenet classification with deep convolutional neural networks. Paper presented at the $25^{\text {th }}$ annual meeting of the Neural Information Processing Systems, Nevada, published as proceedings in Advances in neural information processing systems (pp. 1097-1105). Red Hook, NY: Curran Associates.

Lazareva, O.F., Wasserman, E.A., \& Biederman, I. (2007). Pigeons' recognition of partially occluded objects depends on specific training experience. Perception, 36, 33-48.

Lea, S.E.G., Slater, A.M., \& Ryan, C.M.E. (1996). Perception of object unity in chicks: A comparison with the human infant. Infant Behavior and Development, 19, 501-504.

LeCun, Y., \& Bengio, Y. (1995). Convolutional networks for images, speech, and time series. In M. A. Arbib (Ed.), The handbook of brain theory and neural networks (pp. 276-279). Cambridge, MA: MIT Press. 
Livingstone, M.S., Pettine, W.W., Srihasam, K., Moore, B., Morocz, I.A., \& Lee, D. (2014). Symbol addition by monkeys provides evidence for normalized quantity coding. Proceedings of the National Academy of Sciences, 111, 6822-6827.

Luis, R., Sucar, E., \& Morales, E.F. (2010). Inductive transfer for learning Bayesian networks. Machine Learning, 79, 227-255.

Majaj, N.J., Hong, H., Solomon, E.A., \& DiCarlo, J.J. (2015). Simple learned weighted sums of inferior temporal neuronal firing rates accurately predict human core object recognition performance. Journal of Neuroscience, 35, 13402-13418.

Martin-Malevi, J. (2011). Discrimination of contour-deleted images in baboons (Papio papio) and chimpanzees (Pan troglodytes). Animal Cognition, 14, 415-426.

Matsuzawa, T. (1985). Use of numbers by a chimpanzee. Nature, 315, 57-59.

Minini, L., \& Jeffery, K.J. (2006). Do rats use shape to solve "shape discriminations"? Learning \& Memory, 13, 287-297.

Murofushi, K. (1997). Numerical matching behavior by a chimpanzee (Pan troglodytes): Subitizing and analogue magnitude estimation. Japanese Psychological Research, 39, 140-153.

Nagasaka, Y., Brooks, D.I., \& Wasserman, E.A. (2010). Amodal completion in bonobos. Learning \& Motivation, 41, 174-186.

Nagasaka ,Y., Lazareva, O.F., \& Wasserman, E.A. (2007). Prior experience affects amodal completion in pigeons. Perception \& Psychophysics, 69, 596-605.

Nagasaka, Y., \& Wasserman, E.A. (2008). Amodal completion of moving objects by pigeons. Perception, 37, 557-570.

Nakamura, N., Watanabe, S., Betsuyaka, T., \& Fujita, K. (2011). Do bantams (Gallus gallus domesticus) amodally complete partly occluded lines? An analysis of line classification performance. Journal of Comparative Psychology, 125, 411-419.

Nakayama, K., He, Z.J., \& Shimojo, S. (1995). Visual surface representation: a critical link between lower-level and higher level vision. In S.M. Kosslyn \& D.N. Osherson (Eds.), Invitation to cognitive science (pp. 1-70). Cambridge MA: MIT Press.

Nakayama, K., \& Shimojo. S. (1992). Experiencing and perceiving visual surfaces. Science, 257, $1357-1363$.

Nieder, A. (2002). Seeing more than meets the eye: processing of illusory contours in animals. Journal of Comparative Physiology, A, 188, 249-260. 
Oche, E.S. (2012). Assessing the relative effectiveness of three teaching methods in the measurement of student achievement in mathematics. Journal of Emerging Trends in Educational Research, 3, 479-486.

Patterson, D.K., \& Pepperberg, I.M. (1994). A comparative study of human and parrot phonation: I. Acoustic and articulatory correlates of vowels. Journal of the Acoustical Society of America, 96, 634-648.

Patterson, D.K., \& Pepperberg, I.M. (1998). A comparative study of human and Grey parrot phonation: II. Acoustic and articulatory correlates of stop consonants. Journal of the Acoustical Society of America, 103, 2197-2213.

Peissig, J.J., Young, M.E., Wasserman, E.A., \& Biederman, I. (2005). The role of edges in object recognition by pigeons. Perception, 34, 1353-1374.

Pepperberg, I.M. (1981). Functional vocalizations by an African Grey parrot (Psittacus erithacus). Zeitschrift für Tierpsychologie, 55, 139-160.

Pepperberg, I.M. (1987). Acquisition of the same/different concept by an African Grey parrot (Psittacus erithacus): Learning with respect to categories of color, shape, and material. Animal Learning \& Behavior, 15, 423-432.

Pepperberg, I.M. (1988). An interactive modeling technique for acquisition of communication skills: Separation of 'labeling' and 'requesting' in a psittacine subject. Applied Psycholinguistics, 9, $59-76$.

Pepperberg, I.M. (1992). Proficient performance of a conjunctive, recursive task by an African Grey parrot (Psittacus erithacus). Journal of Comparative Psychology, 106, 295-305.

Pepperberg, I.M. (1994). Evidence for numerical competence in an African Grey parrot (Psittacus erithacus). Journal of Comparative Psychology, 108, 36-44.

Pepperberg, I.M. (1999). The Alex studies: Cognitive and communicative abilities of Grey parrots. Cambridge, MA: Harvard.

Pepperberg, I.M. (2006). Addition by a Grey Parrot (Psittacus erithacus), including absence of quantity. Journal of Comparative Psychology, 120, 1-11.

Pepperberg, I.M., \& Brezinsky, M.V. (1991). Acquisition of a relative class concept by an African Grey Parrot (Psittacus erithacus): Discriminations based on relative size. Journal of Comparative Psychology, 105, 286-294.

Pepperberg, I.M., \& Carey, S. (2012). Grey parrot number acquisition: The inference of cardinal 
value from ordinal position on the numeral list. Cognition, 125, 219-232.

Pepperberg, I.M., \& Gordon, J. (2005). Numerical comprehension by a Grey parrot (Psittacus erithacus), including a zero-like concept. Journal of Comparative Psychology, 119, 197-209.

Pepperberg, I.M., \& Hartsfield, LA. (2014) Do Grey parrots (Psittacus erithacus) succeed on a "complex" foraging task failed by primates but solved by fish? Journal of Comparative Psychology, 128, 298-306. doi: 10.1037/a0036205.

Pepperberg, I.M., \& Shive, H.R. (2001). Simultaneous development of vocal and physical object combinations by a Grey parrot (Psittacus erithacus): Bottle caps, lids, and labels. Journal of Comparative Psychology, 115, 376-384.

Pepperberg, I.M., \& Wilcox, S.E. (2000). Evidence for mutual exclusivity during label acquisition by Grey parrots (Psittacus erithacus)? Journal of Comparative Psychology, 114, 219-231.

Pepperberg, I.M., \& Wilkes, S. (2004). Lack of referential vocal learning from LCD video by Grey parrots (Psittacus erithacus). Interaction Studies, 5, 75-97.

Pepperberg, I.M., Koepke, A., Livingston, P., Girard, M., \& Hartsfied, L.A. (2013). Reasoning by inference: Further studies on exclusion in Grey parrots (Psittacus erithacus). Journal of Comparative Psychology, 127, 272-281. doi: 10.1037/a0031641

Pepperberg, I.M., Vicinay, J., \& Cavanagh, P. (2008). The Müller-Lyer illusion is processed by a Grey parrot (Psittacus erithacus). Perception, 37, 765-781.

Pepperberg, I.M., Willner, M.R., \& Gravitz, L.B. (1997). Development of Piagetian object permanence in a Grey parrot (Psittacus erithacus). Journal of Comparative Psychology, 111, 63-75.

Prior, H., \& Güntürkün, O. (1999). Patterns of visual lateralization in pigeons: Seeing what is there and beyond. Perception, 28(suppl), 22.

Prior, H., \& Wilzeck, C. (2008). Selective feeding in birds depends on combined processing in the left and right brain hemisphere. Neuropsychologia, 46, 233-240.

Premack, D. (1976). Intelligence in ape and man. Hillsdale, NJ: Lawrence Erlbaum Associates.

Regolin, L., \& Vallortigara, G. (1995). Perception of partly occluded objects in young chicks. Perception and Psychophysics, 57, 971-976.

Regolin, L., Marconato, F., \& Vallortigara, G. (2004). Hemispheric differences in the recognition of partly occluded objects by newly hatched domestic chicks (Gallus gallus). Animal Cognition, 7, 162-170. 
Rozin, P. (1976). The evolution of intelligence and access to the cognitive unconscious. In J.M. Sprague \& A.N. Epstein (Eds.), Progress in psychobiology and physiological psychology, Vol. 6 (pp. 245-280). New York: Academic Press.

Sato, A., Kanazawa, S., \& Fujita, K. (1997). Perception of object unity in a chimpanzee (Pan troglodytes). Japanese Psychological Research, 39, 191-199.

Sekuler, A.B., Lee, J.A.J., \& Shettleworth, S.J. (1996). Pigeons do not complete partly occluded figures. Perception, 25, 1109-1120.

Stephan, C., Wilkinson, A., \& Huber, L. (2013) Pigeons discriminate objects on the basis of abstract familiarity. Animal Cognition, 16, 983-992.

Todt, D. (1975). Social learning of vocal patterns and modes of their applications in Grey Parrots. Zeitschrift für Tierpsychologie, 39, 178-188.

Tvardíková, K., \& Fuchs, R. (2010). Tits use amodal completion in predator recognition: a field experiment. Animal Cognition, 13, 609-615.

Ushitani, T., \& Fujita, K. (2005) Pigeons do not perceptually complete partially occluded photos of food: an ecological approach to the 'pigeon problem'. Behavioural Processes, 69, 67-78.

Ushitani, T., Fujita, K., \& Yamanaka, R. (2001). Do pigeons (Columba livia) perceive object unity? Animal Cognition, 4, 153-161.

Vallortigara, G. (2006) The cognitive chicken: Visual and spatial cognition in a non-mammalian brain. In E.A. Wasserman \& T.R. Zentall (Eds.), Comparative cognition: Experimental explorations of animal intelligence (pp 53-70). Oxford: Oxford University Press.

Vallortigara, G., Snyder, A., Kaplan, G., Bateson, P., Clayton, N., \& Rogers, L.J. (2008). Are animals autistic savants? PLoS Biology, 6 (2) e42.

Van Hateren, J.H., Srinivasan, M,V., \& Wait, P.B. (1990). Pattern recognition in bees: orientation discrimination. Journal of Comparative Physiology A, 167, 649-654.

von der Heydt, R., Peterhans, E., \& Baumgartner, G. (1984). Illusory contours and cortical neuron responses. Science, 224, 1260-1262.

Wasserman, E.A., \& Zentall, T.R. (Eds.) (2006). Comparative cognition: Experimental explorations of animal intelligence. New York: Oxford University Press.

Wilkie, S.E., Vissers, P.M.A.M., Das, D., DeGrip, W.J., Bowmaker, J.K., \& Hunt, D.M. (1998). The molecular basis for UV vision in birds: spectral characteristics, cDNA sequence and retinal localization of the UV-sensitive visual pigment of the budgerigar (Melopsittacus undulatus). 
Biochemical Journal, 330, 541-547.

Zanforlin, M. (1981). Visual perception of complex forms (anomalous surfaces) in chicks. Italian Journal of Psychology, 8, 1-16.

Zhou, H., Friedman, H. S., \& Von Der Heydt, R. (2000). Coding of border ownership in monkey visual cortex. The Journal of Neuroscience, 20(17), 6594-6611.

Zentall, T.R., \& Wasserman, E.A. (Eds) (2012). The Oxford handbook of comparative cognition. New York: Oxford University Press. 


\section{Figure Legends}

Figure 1. (a) occluded and (b) illusory objects.

Figure 2. Samples of 3D objects used to train Griffin on shapes. Objects are made of wood, plastic, and wool (felt).

Figure 3. Griffin in an M/R session.

Figure 4. A 'dummy' occulusion stimulus. Blue object was the target of the question ("What shape blue?'). Colors and shapes varied over trials.

Figure 5. Samples of Kaniza figures, occluded figures, and probes presented to Griffin. Numbers are expected "x-corner" responses.

Figure 6. Schematic of testing format. Questioner is on one side of Griffin, holding the stimulus on the other. On-line listerner/evaluator, blind to the stimulus, is at the other end of the laboratory room. The question to Griffin is "What shape blue?"; his response is "3-corner."

Figure 7. Percentages correct for modal and amodal completion tasks and amodal probes. Chance conservatively configured at $1 / 5$, for the labels needed to respond to the stimuli.

Figure 8. Correct responses are in diagonal boxes, incorrect responses are in off-diagonal boxes. Numbers inside objects represent number of times that object was tested. Objects were of various sizes and colors. The single shaded object (cell 4,4) represents the single 3D object presented to Griffin.

Figure 9. Correct responses are in diagonal boxes, incorrect responses are in off-diagonal boxes. Numbers inside objects represent number of times that object was tested. Objects, pacmen, and circles were of various sizes and objects were of various colors.

Figure 10. Possible process whereby over-learned 3D objects can be recognized in 2D depictions. (a) 3D obects seen separated, no occlusion. (b) 3D objects can be sampled as 2D images in the real world. 
${ }^{1}$ One exception to normative probes existed: Students in the laboratory, when asked the shape of the object with an appropriate piece removed from a hexagon, continued to label it "6-corner", even though it now had eight corners. (They had counted the corners of the other detached probes appropriately.) We thus hesitated to use it as a stimulus for Griffin. Instead, we used a pentagon-a shape on which Griffin had not been trained—with a displaced occluder, under the assumption that if Griffin labeled it "6-corner", he would again be counting the corners of an unfamiliar object.

${ }^{2}$ Specifically, colors such as orange and purple - which can be problematic for Greys to identify (Pepperberg, 1994, 2006) because parrots' abilities to see in the UV cause their color perception to differ somewhat from that of humans (Bowmaker et al., 1994, 1996; Carvalho et al., 2012; Cuthill et al., 2000; Goldsmith \& Butler, 2005; Wilkie et al., 1998)—were adjusted within appropriate ranges. 


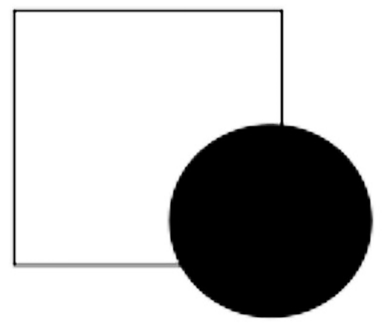

(a)

Figure 1
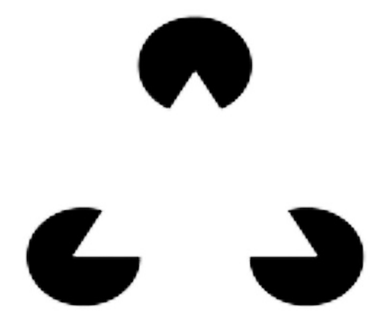

(b) 


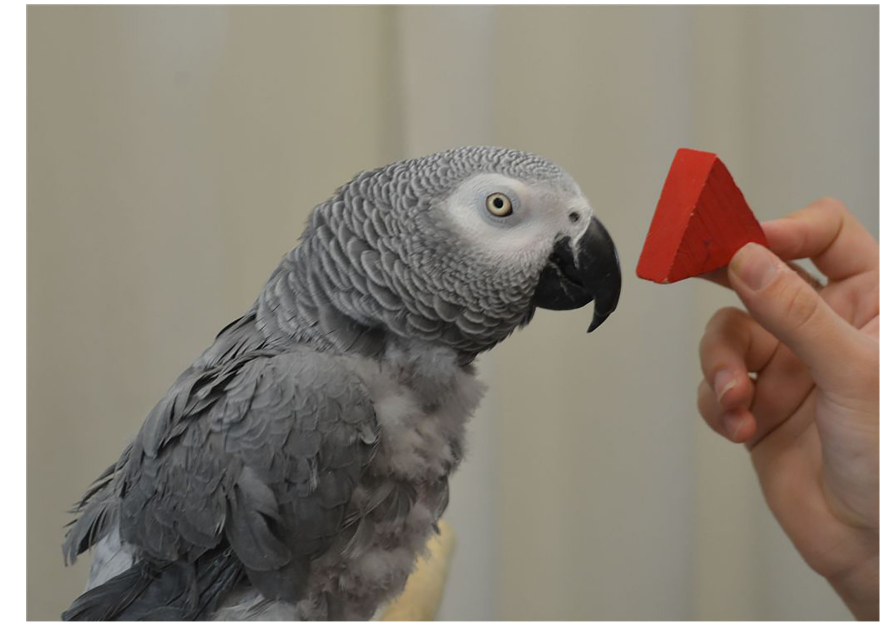

Figure 3

$$
3
$$

3

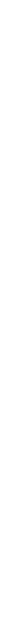


Figure 4
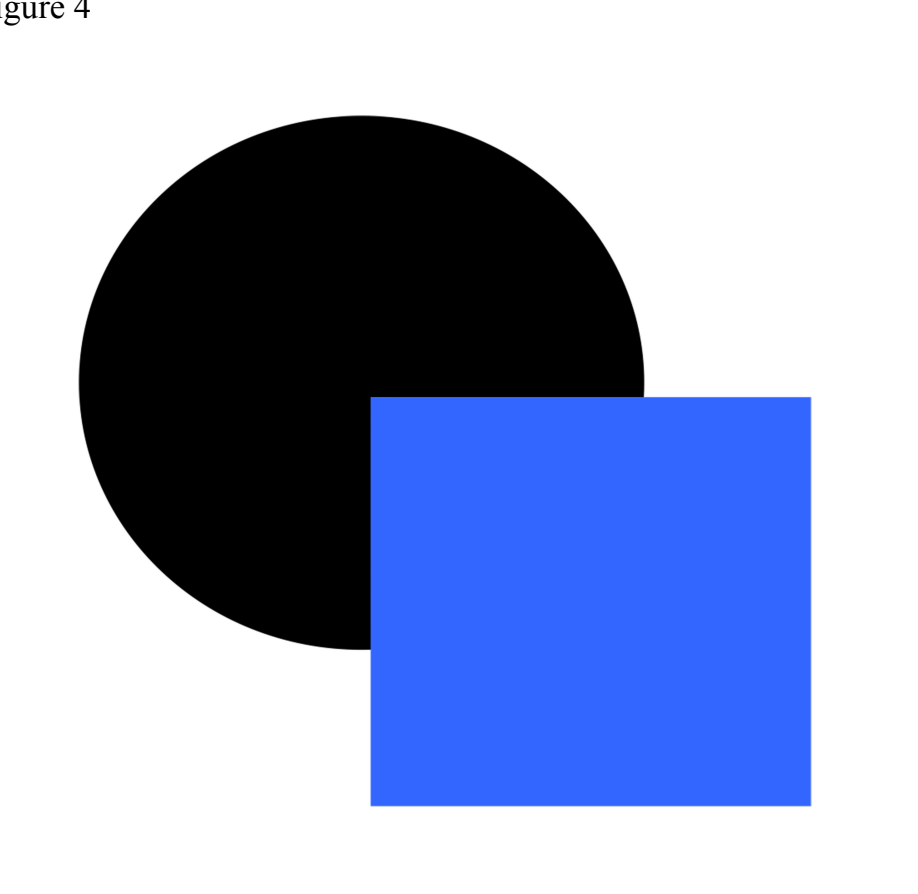
(a)

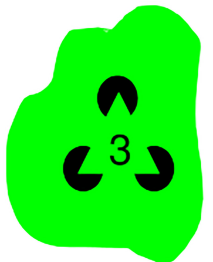

(e)

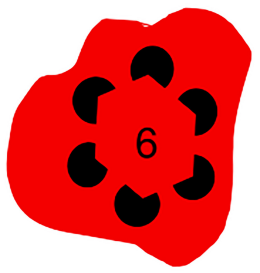

(i)

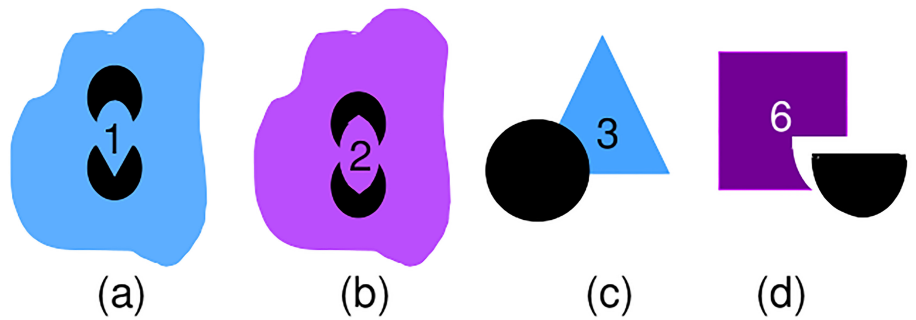

(b)

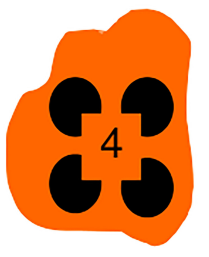

(f)

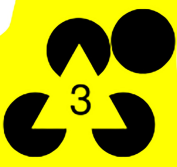

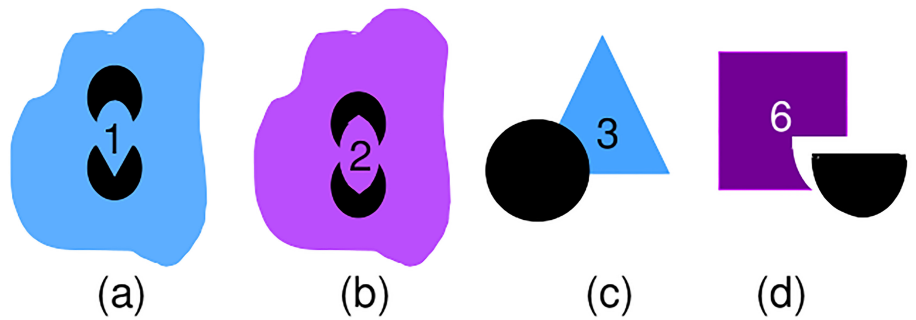

(c)

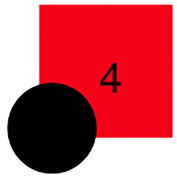

(g)

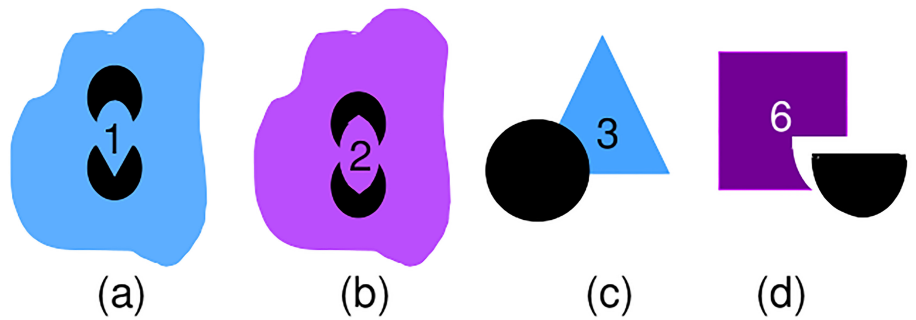

(d)

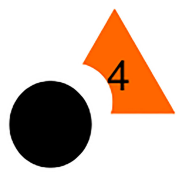

(h)

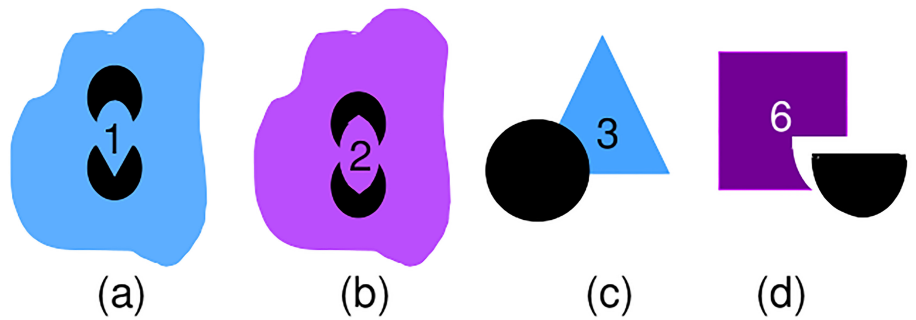

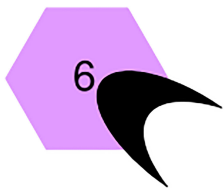

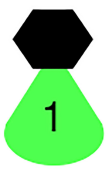
(j)
(k)
(I)
)
) 
Figure 6

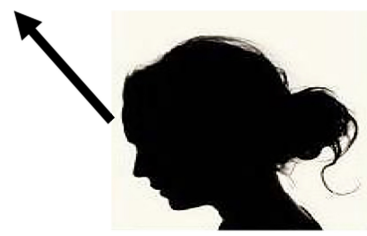

\section{On-line \\ listener/evaluator}

Questioner

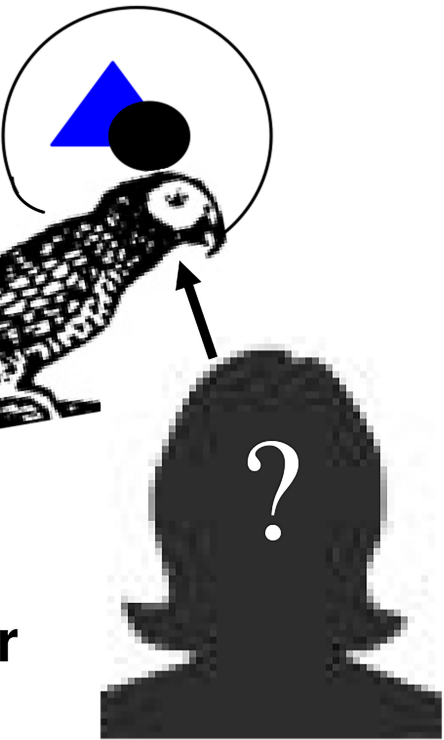




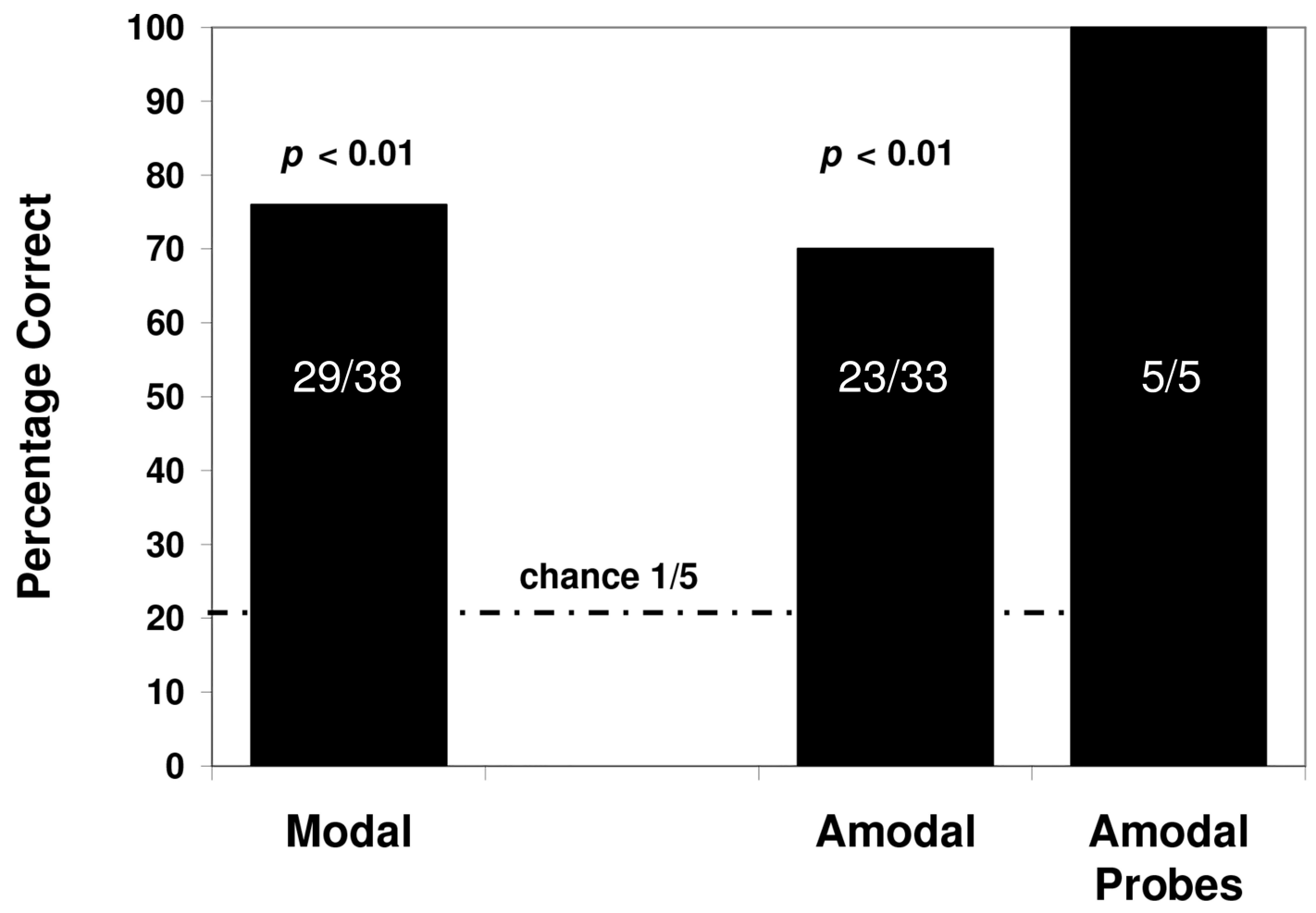


Amodal Completion Shown to Griffin

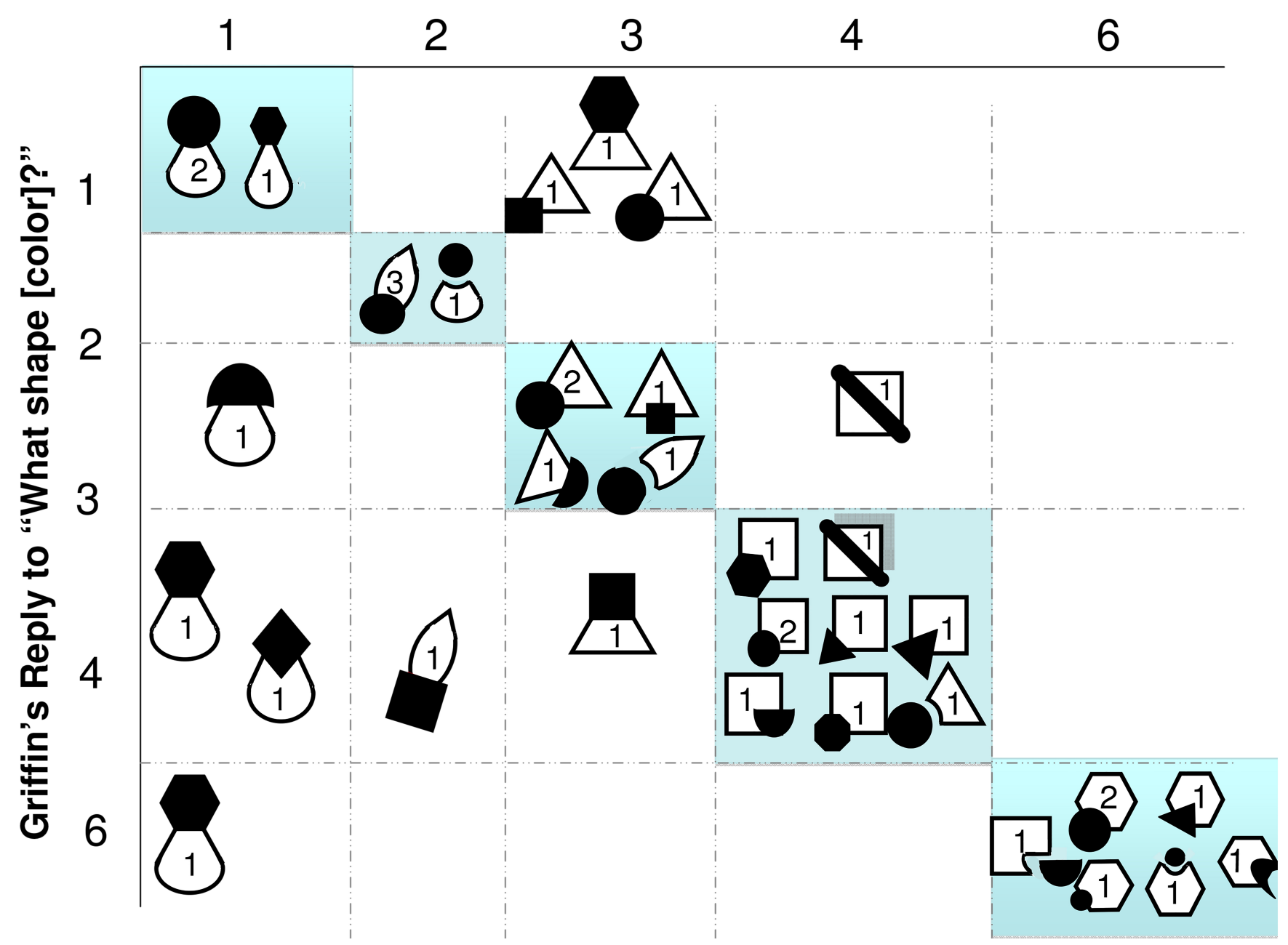


Modal Completion (subjective figure) Shown to Griffin

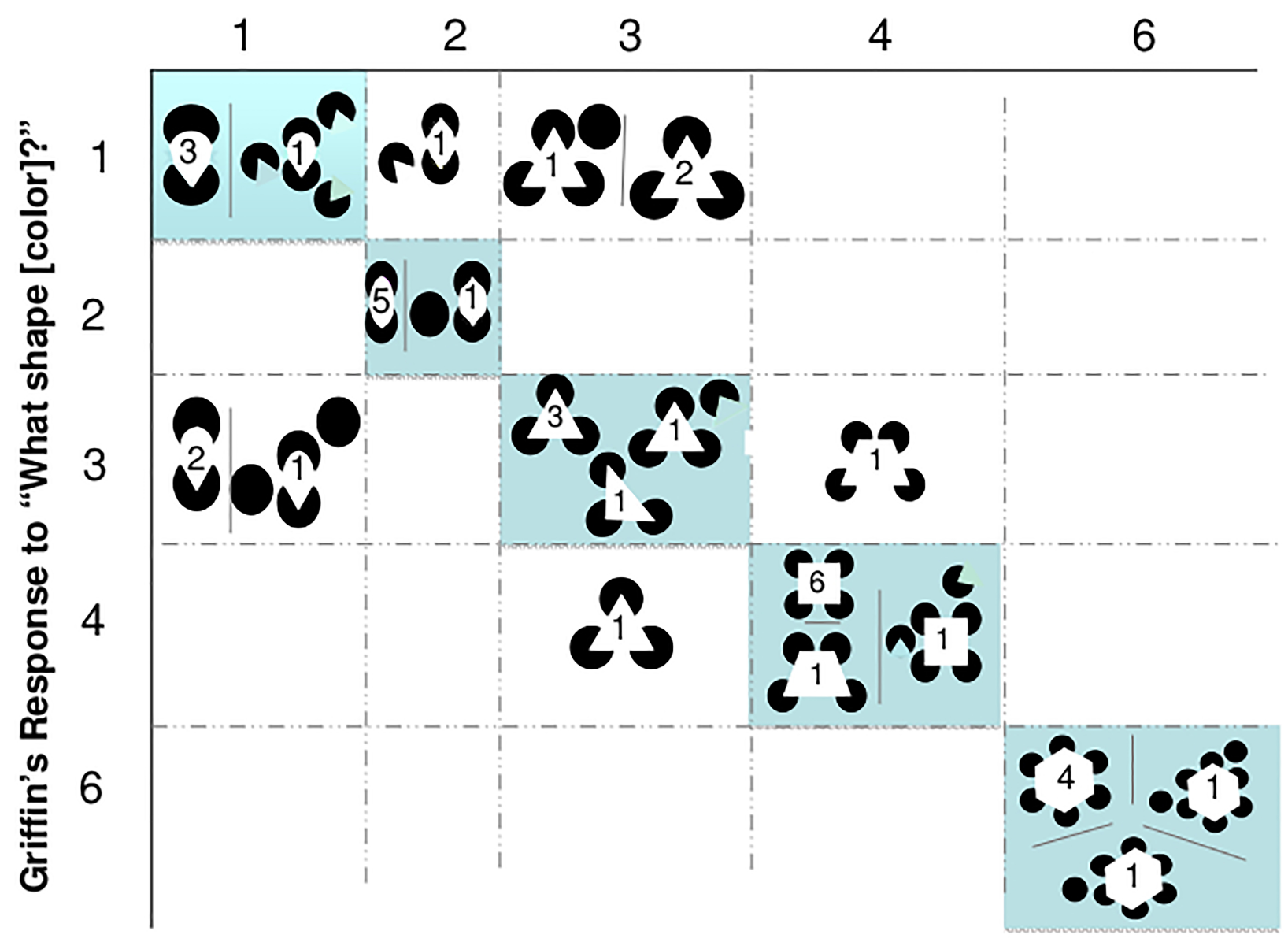


